\title{
The Effect of Acute Inpatient Rehabilitation on Functional Outcomes and Discharge Disposition Following Liver Transplant
}

\author{
Meghan Willoughby ${ }^{1}$, Jacob Ramsey-Morrow ${ }^{1}$, Kyle Littell ${ }^{2}$ \\ 1. Indiana University School of Medicine, Indianapolis, Indiana \\ 2. Indiana University School of Medicine; Rehabilitation Hospital of Indiana, Indianapolis, \\ Indiana
}

Background: Patients undergoing liver transplantation often face many challenges with functional independence, which acute rehabilitation can assist them in overcoming. Due to increased yearly liver transplantations, further investigation is required to evaluate the efficiency of acute rehabilitation. The objective of this study is to analyze the efficacy of acute rehabilitation in patients who underwent liver transplantation, primarily using Functional Independence Measure $\left(\mathrm{FIM}^{\mathrm{TM}}\right)$ scores and discharge disposition.

Methods: A retrospective chart review was conducted on 143 encounters, consisting of 107 patients who underwent liver transplantation. Inclusion factors consisted of undergoing liver transplantation between January 2014-December 2018, admission into acute rehabilitation within 6 months post-transplant, and the availability of admission date, admission $\mathrm{FIM}^{\mathrm{TM}}$, duration of stay, discharge $\mathrm{FIM}^{\mathrm{TM}}$, and discharge disposition. These factors were evaluated in this study.

Results: Patients who underwent acute rehabilitation following liver transplantation were found to have statistically significant positive FIM $^{T M}$ change $(P<0.00001)$ and $\mathrm{FIM}^{\mathrm{TM}}$ efficiency $(\mathrm{P}<$ 0.00001 ). The mean $\mathrm{FIM}^{\mathrm{TM}}$ change and efficiency were $25.4 \pm 18.5$ and $2.0 \pm 1.6$, respectively, for patients meeting inclusion criteria, and $35.7 \pm 11.8$ and $2.4 \pm 1.0$, respectively, when return to acute care (RTAC) encounters were excluded. A positive correlation was found between longer duration of stay in acute rehabilitation and positive FIM $^{\mathrm{TM}}$ change for all patients meeting inclusion criteria $(P<0.00001, r=0.465)$, and excluding RTAC encounters $(P<0.00001, r=$ 0.393). $34.3 \%(n=49)$ of encounters had an RTAC, 3.5\% $(n=5)$ were discharged to a skilled nursing facility (SNF), and 62.2\% ( $n=89)$ were discharged to the community. Overall, $83.2 \%(n$ = 89) of patients ultimately had a community discharge. Infection, respiratory/CV complications, and gastrointestinal complications were the most common causes for RTAC.

Conclusion: Acute rehabilitation provides patients who have received a liver transplant with the opportunity to significantly improve their function and independence. 